\title{
Abnormal most-rapid isometric contractions in patients with Parkinson's disease
}

\author{
M M Wierzbicka, A W Wiegner, E L Logigian, R R Young
}

\begin{abstract}
Fast isometric elbow flexor muscle contractions of specified amplitude in six normal subjects were compared with those of 11 patients with Parkinson's disease. Despite treatment, all patients exhibited deficits in this motor task. Three patients were able to produce rapid force pulses with normal contraction times, but the variability of their force responses was increased in comparison with the highly stereotyped responses produced by normal subjects. The other eight patients had prolonged contraction times and segmentation of the force profiles. The integrated area of the first agonist EMG burst and the rate of development of force (dF/dt) were less at any target level than what was needed to produce a fast response. The area of the EMG burst, however, did increase with target amplitude, and the relative increase of $\mathrm{dF} / \mathrm{dt}$, with target amplitude, was normal. It is concluded that the motor program subserving fast muscle contraction is preserved in Parkinson's disease, but its execution is characterised by improper scaling of motor output.
\end{abstract}

Bradykinesia, or slowness of voluntary movement including its initiation, is a characteristic feature of Parkinson's disease (PD). A number of studies of single joint, goal directed movements in patients with PD have used movement time as a quantitative indicator of Parkinsonian bradykinesia. ${ }^{1-6}$ Improvement of movement velocity has been seen in patients treated with levodopa ${ }^{57}$ although, in general, velocity remains slower than normal; and performance of a rapid single-joint movement task does not improve as much with drug therapy as does overall mobility in daily life. ${ }^{7}$

Although many investigators have found movement time to be prolonged in patients with PD, it is still not clear what mechanisms account for this phenomenon. Flowers ${ }^{1}$ showed that patients with PD have particular difficulty in performing large amplitude movements in normal time. Hallett and Khoshbin $^{4}$ hypothesised that bradykinesia results from insufficient muscle energy, that the initial agonist electromyographic (EMG) burst starting a movement cannot be increased sufficiently to produce rapid movement of required amplitude and thus additional EMG bursts are needed. Conversely, Berardelli et $\mathrm{al}^{7}$ recently reported that although patients moved slowly, the absolute size and duration of the first agonist EMG burst, and the percentage increase in the amplitude of the first agonist burst for movements of a different size (or agonist load), were similar in patients and in normal subjects. The authors concluded that the agonist burst size was "inappropriately scaled to the movement amplitude and velocity". This implies that Parkinsonian patients need to produce larger agonist bursts than normal subjects to achieve movements with normal velocity. Berardelli et al ${ }^{7}$ suggested that one possible explanation of such results would be an abnormality in the force-EMG relation in the muscles of patients with PD.

In this paper we investigate this hypothesis further by comparing the rapid development of force in normal subjects and patients with PD. Since other studies have reported that rapid isotonic arm movements produced by PD patients are often mechanically smooth, despite discrete bursts in the agonist EMG pattern, ${ }^{45}$ we chose an isometric paradigm to minimise the smoothing effect of arm inertia and accentuate the underlying irregularities in muscle force. Quantitative analysis of force responses and electromyographic (EMG) activity from the antagonistic muscle pair (biceps, triceps) was performed.

\section{Methods}

Most-rapid targeted force pulses were studied in 11 patients with PD (mean age 64 years, range 51-83 years) and six normal subjects (mean age 60 years, range 40-75 years). Patients chosen for this study were mildly or moderately affected, as those with severe disease were unable to perform the test. All patients participating in the study were on their normal daily dosage of anti-Parkinsonian medication and were tested when their medication was most effective, so they had relatively little clinical evidence of bradykinesia or rigidity and virtually no tremor at the time of the test. The clinical stage and drug regime of each patient are given in the table. The dominant arm was usually tested, but when there was a significant difference between the two sides, the more affected arm was studied.

During the test, subjects were seated in a comfortable chair with the shoulder abducted and elbow flexed at 90 degrees. The semipronated forearm was immobilised in a rigid plastic splint attached to a table by a load cell which measured force developed at the elbow. 
Table Summary data from normal and Parkinsonian subjects. The latter are divided as shown into Group 1 (top 3) and Group 2 (remainder) based on MVC and movement speed

\begin{tabular}{|c|c|c|c|c|c|c|c|}
\hline & \multicolumn{4}{|c|}{ Normal Subjects } & & & \\
\hline & Subject & Age & Sex & $\begin{array}{l}M V C \\
\left(\mathrm{~kg}^{a}\right.\end{array}$ & & & \\
\hline & $\begin{array}{l}1 \\
2 \\
3 \\
4 \\
5 \\
6 \\
\text { Mean }\end{array}$ & $\begin{array}{l}40 \\
50 \\
53 \\
71 \\
74 \\
75 \\
60\end{array}$ & $\begin{array}{l}\mathbf{M} \\
\mathbf{M} \\
\mathbf{M} \\
\mathbf{M} \\
\mathbf{F} \\
\mathbf{M}\end{array}$ & $\begin{array}{r}32 \cdot 8 \\
30.4 \\
31.9 \\
16 \cdot 3 \\
9 \cdot 0 \\
17 \cdot 4\end{array}$ & & & \\
\hline & \multicolumn{7}{|c|}{ Parkinson's Disease } \\
\hline & Subject & Age & Sex & $\begin{array}{l}M V C \\
(\mathrm{~kg})\end{array}$ & Stage $^{b}$ & $\begin{array}{l}\text { Duration } \\
\text { (year) }\end{array}$ & Therapy \\
\hline$\underset{1}{\text { Group }}$ & $\begin{array}{l}1 \\
2 \\
3\end{array}$ & $\begin{array}{l}56 \\
59 \\
75\end{array}$ & $\begin{array}{l}\mathbf{M} \\
\mathbf{M} \\
\mathbf{M}\end{array}$ & $\begin{array}{l}26 \cdot 5 \\
25 \cdot 2 \\
26 \cdot 1\end{array}$ & $\begin{array}{l}\text { II } \\
\text { II } \\
\text { II }\end{array}$ & $\begin{array}{r}10 \\
2 \\
3\end{array}$ & $\begin{array}{l}\text { Sinemet } 150 / 1500 \mathrm{mg} \\
\text { Cogentind } 6 \mathrm{mg} \\
\text { Artane }^{\mathrm{c}} 12 \mathrm{mg}\end{array}$ \\
\hline$\underset{2}{\text { Group }}$ & $\begin{array}{c}4 \\
5 \\
6 \\
7 \\
8 \\
9 \\
10 \\
11 \\
\text { Mean }\end{array}$ & $\begin{array}{l}51 \\
59 \\
59 \\
63 \\
65 \\
65 \\
66 \\
83 \\
64\end{array}$ & $\begin{array}{l}\mathbf{M} \\
\mathbf{M} \\
\mathbf{F} \\
\mathbf{M} \\
\mathbf{M} \\
\mathbf{M} \\
\mathbf{F} \\
\mathbf{M}\end{array}$ & $\begin{array}{l}18 \cdot 7 \\
15 \cdot 0 \\
15 \cdot 9 \\
15 \cdot 1 \\
19 \cdot 2 \\
19 \cdot 4 \\
16 \cdot 5 \\
12 \cdot 6\end{array}$ & $\begin{array}{l}\text { IV } \\
\text { III } \\
\text { I } \\
\text { III } \\
\text { III } \\
\text { II } \\
\text { II } \\
\text { II }\end{array}$ & $\begin{array}{r}15 \\
14 \\
6 \\
20 \\
8 \\
8 \\
10 \\
6\end{array}$ & $\begin{array}{l}\text { Sinemet 60/600, Parlodel' } 20 \mathrm{mg} \\
\text { Sinemet } 50 / 500, \text { Parlodel } 10 \mathrm{mg} \\
\text { Artane } 12 \mathrm{mg} \\
\text { Sinemet } 75 / 750, \text { Parsidol }^{8} 150 \mathrm{mg} \\
\text { Sinemet } 75 / 750 \\
\text { Sinemet } 75 / 750 \\
\text { Sinemet } 112 / 450, \text { Artane } 6 \mathrm{mg} \\
\text { Sinemet } 112 / 450\end{array}$ \\
\hline
\end{tabular}

"Maximum voluntary contraction; ${ }^{b}$ Hoehn and Yahr scale evaluation off medicine; ccarbidopa/levodopa; ${ }^{d}$ benztropine mesylate; 'trihexyphenidyl hydrochloride; 'bromocriptine mesylate; "ethopropazine hydrochloride.

A storage oscilloscope, facing the subject, displayed both target force as a horizontal line and the actual force-time trajectory produced by the subject. Subjects were asked to generate a "brief, rapid force pulse" whose peak amplitude matched a given target force. Subjects were urged to respond to the target step "when ready" rather than "as soon as possible". A two second period, defined by a two second audible tone together with the appearance of the target on the oscilloscope screen, was allowed for each response. The arm, immobilised by the splint, did not move. Responses were initiated from a resting force level.

Subjects were allowed sufficient practice trials to familiarise themselves with the apparatus. Force responses were acquired in blocks of 20 trials, followed by a few minutes of rest. Within each block, the target amplitude was constant. Targets of 4,8 , and $12 \mathrm{~kg}$ were given in randomised order. (One control subject was unable to produce $12 \mathrm{~kg}$ responses and was tested only at 4 and $8 \mathrm{~kg}$.) In addition, at the beginning of the experiment each subject's maximum voluntary contraction (MVC) of the elbow flexors was measured as an average over a two second interval. EMG was recorded from the biceps muscle and lateral head of triceps with Beckman bipolar surface electrodes. EMG signals were preamplified and filtered with a bandpass of $8 \mathrm{~Hz}-8 \mathrm{kHz}$. Presentation of targets and data acquisition were controlled by a PDP 11/ 23 computer. Force and EMG were each sampled at $500 \mathrm{~Hz}$ and stored for further offline analysis.

When analysed, EMG signals were rectified and passed through a 9-point least squares smoothing filter. The onset and termination of EMG bursts were marked manually using an interactive cursor program, and EMG during each burst was integrated. Force rise time was computed automatically as the interval between when developed force reached $5 \%$ of maximal force, and maximal force. Actual force rise times were thus slightly underestimated by our automatic routine, which was chosen for its consistency, in preference to manual measurements of the onset of force, which would be subject to random error and possible observer bias. The rate of change of force $(\mathrm{dF} / \mathrm{dt})$ was obtained by numerical differentiation of the force signal and the peak was identified using a cursor. Because differences in arm stiffness, ${ }^{8}$ electrode placement, or skin resistance can make it difficult to compare EMG burst parameters between subjects, comparisons were limited to data from the same subject. Linear regression analysis and the student's $t$ test for uncorrelated means were used for statistical evaluation of the results.

\section{Results}

Figure 1 shows several force responses of two normal subjects, to a $12 \mathrm{~kg}$ target, aligned at the onset of force. All normal subjects performed fast elbow flexions with a single sequence of EMG bursts from agonist and antagonist muscles. Four subjects typically had a reciprocal pattern of EMG bursts similar to that observed in ballistic movements (fig 1A), whereas the other two subjects showed almost synchronous activation of biceps and triceps (fig 1B). Note also the smooth and highly stereotyped force trajectories and consistent shape of the $\mathrm{dF} / \mathrm{dt}$ curves. The average force rise time of six subjects was $69(16) \mathrm{ms}$, mean (SD), as seen in fig 2 . In individual subjects the contraction 
time varied relatively little with peak force: the slope of the regression line for contraction time versus peak force ranged from $-1 \cdot 3$ $\mathrm{ms} / \mathrm{kg}$ to $+2 \cdot 8 \mathrm{~ms} / \mathrm{kg}$ and was not correlated with MVC (correlation coefficient $r=0 \cdot 16$, $\mathrm{p}>0.05)$. Pooling all data from control subjects, force rise time increased significantly with peak force $(r=0.15, p<0.05$, fig $3 \mathrm{~A})$. The slope of this relationship was small, only $0.7 \mathrm{~ms} / \mathrm{kg}$, because normal subjects were able to scale their contraction speed, that is, peak $\mathrm{dF} / \mathrm{dt}$, proportionally to the increase in target amplitude (fig 4).

Patients were divided a posteriori into two groups, based on their MVC and ability to make brief force pulses.

The three patients with highest MVC (Group 1, table) produced contractions with brief force rise times [72 (26) ms], not statistically different from those recorded in normal subjects ( $t$ test, $\mathrm{p}>0.05$, fig 2 ). These force responses, however, were more variable and less smooth, ranging from essentially normal to occasionally grossly abnormal (fig 5). Variability of force trajectories was quantified using the coefficient of variation ( $\mathrm{SD} / \mathrm{mean}$ ) of the peak force and force rise time. In these three patients, coefficients of variation of both force rise time (fig 6A) and peak force (fig 6B) were usually beyond the two standard deviation limit obtained from normal subjects. Nevertheless, patients in this group still scaled their contraction speed $(\mathrm{dF} / \mathrm{dt})$ according to the target amplitude, as did normal subjects (fig 4) so that the contraction time remained approximately constant and in most trials within normal limits (fig 3B). The slope of the regression line for rise time versus maximal force
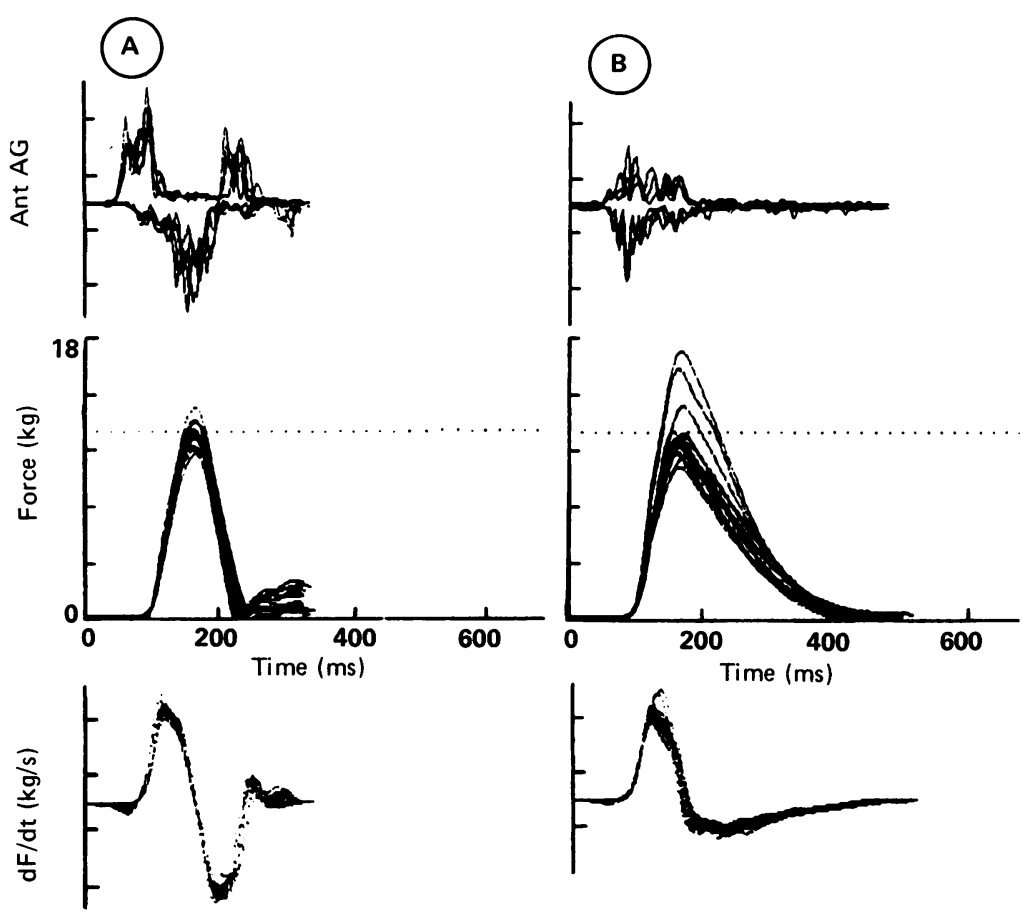

Figure 1 Most rapid force responses aimed at a $12 \mathrm{~kg}$ target in two normal subjects, superimposed on the basis of the time at which force increased above the baseline (middle); rectified and smoothed EMG recorded from biceps and triceps (top); first time derivative of force $(d F / d t$, bottom). Note the differences in subjects' EMG pattern ( $A$, reciprocal, versus $B$, co-contraction) and resulting shape of the force trajectories.

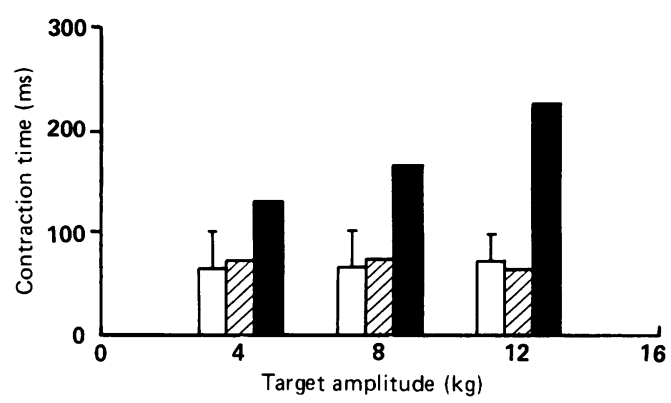

Figure 2 Comparison of mean contraction time for different target amplitudes in normal subjects (empty bar), Group 1 patients (lightly hatched bar), and Group 2 patients (solid bar). Vertical bars indicate $\pm 2 S D$ for normal subjects.

ranged from $-1.8 \mathrm{~ms} / \mathrm{kg}$ to $1.0 \mathrm{~ms} / \mathrm{kg}$. The EMG pattern (fig 5) was similar to that recorded in normal subjects, with alternating bursts in antagonistic muscles observed in one subject, and a coactivation pattern seen in the other two subjects.

The force responses of eight patients with smaller MVC (Group 2, table) were abnormally segmented (fig 7), with force rise times prolonged to 171 (71) $\mathrm{ms}$ (fig 2) and more strongly dependent on target amplitude (fig 2, 3C). In all but one patient, the slope of the regression line for rise time versus maximal force was much larger (range $0.8-24 \mathrm{~ms} / \mathrm{kg}$, mean $13 \mathrm{~ms} / \mathrm{kg}$ ) than in normal subjects. This slope, for all patients, was strongly correlated with MVC ( $\mathrm{r}=-0.83, \mathrm{p}<0.01)$. Patients in Group 2 had lower mean $\mathrm{dF} / \mathrm{dt}$ than normal subjects for all target amplitudes (fig 4). However, the percentage increase of $\mathrm{dF} / \mathrm{dt}$ with target amplitude was similar to that observed in normal subjects. This can be seen in fig 4 , in which the dependence of $\mathrm{dF} / \mathrm{dt}$ on peak force can be approximated by a straight line through the origin for both groups. Variability of force rise time and peak force,

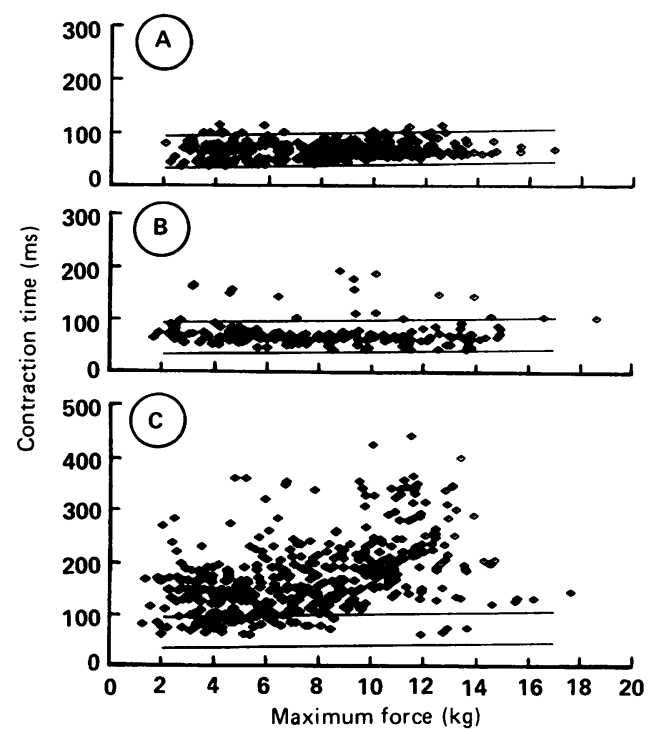

Figure 3 Force rise time (contraction time) as a function of maximum force achieved. $A=$ normal subjects, $B=$ Group 1 patients, $C=$ Group 2 patients. Each symbol represents a single trial. Solid lines are $95 \%$ confidence limits for normal subjects. 
Figure 4 Maximum contraction speed (peak $d F / d t$ ) as a function of maximum force achieved for each target. Mean values are compared in normal subjects (squares), Group 1 patients (triangles), and Group 2 patients (circles). Error bars indicate $\pm 2 S D$ from the mean for normals.

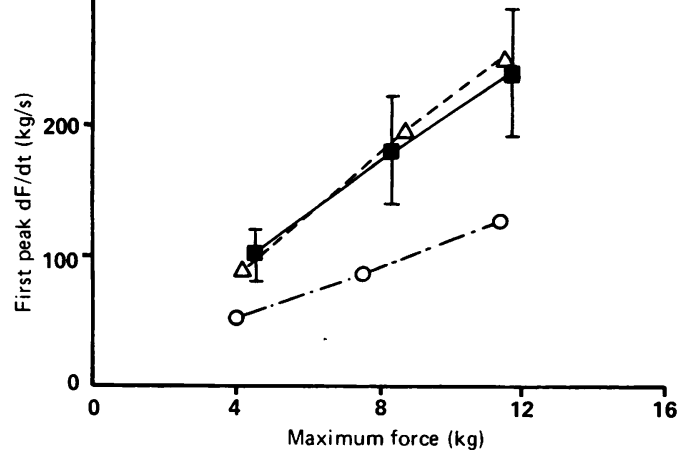

quantified with the coefficient of variation, was smaller than for patients in Group 1 (fig 6): thus, each patient in Group 2 produced abnormal but fairly consistent responses, whereas responses of patients in Group 1 varied from almost normal to grossly impaired.

In most Group 2 patients, EMG indicated coactivation of biceps and triceps. The EMG pattern was composed of either more than one burst of EMG activity, each approximately of the same duration, separated by clear silent periods (fig 7A), or prolonged bursts of agonist EMG activity without silent periods (fig 7B). Since it was difficult to identify individual bursts in this latter EMG pattern, as well as the resultant corresponding steps in the force response, we restricted our quantitative analysis of the EMG-force relationship to two patients who consistently produced clearly segmented EMG bursts.

Analysis of the force profiles in these two patients showed that the individual step-like contractions within the overall force profile were relatively fast. The first peak of $\mathrm{dF} / \mathrm{dt}$, plotted against the peak force reached at the end of the first step, was similar to that recorded in normal subjects (fig 8A). (We will make use of this fairly "normal" result below.) However, if the first peak of $\mathrm{dF} / \mathrm{dt}$ is plotted versus maximal force, at the peak of the last step, speed is, of course, seen to be below that developed by normal subjects, and fig $\mathbf{8 B}$ indicates the degree to which this occurs, especially at high peak forces.

In a normal subject, the EMG burst area for larger targets ( 8 and $12 \mathrm{~kg}$ ) can be predicted from the regression line fitted to data from the $4 \mathrm{~kg}$ target (fig 9A). Since we have chosen not to compare absolute values of EMG from subject to subject, we have a problem in choosing the "gold standard" for the EMG-force relation in patients. Referring back to fig $8 \mathrm{~A}$, we see that the speed of the first force segment, resulting from the first agonist EMG burst, was essentially normal. If we plot the first EMG burst area against the force which it produced (fig 9B), we can "calibrate" the EMG-force relation for this patient, based on the initial fast portion of each response. Note that the range of forces produced in fig 9A (filled circles) and fig $9 \mathrm{~B}$ are similar, although 8 and $12 \mathrm{~kg}$ targets are included in fig 9B. Thus the regression line in fig $9 B$ reflects the EMG-force relation which we would expect to see whenever this patient made fast movements. If we plot the initial EMG burst areas versus the actual force ultimately reached (fig 9C), however, the EMG produced is seen to be approximately half that which would be required for fast movements to the target. This quantitative analysis of the first segmented response leads to the conclusion that the magnitude of the first agonist EMG burst is insufficient to reach the target, and that this mismatch becomes increasingly large for larger targets.
Figure 5 Fast contractions produced by a patient in Group 1. (Top) EMG recorded from biceps and triceps. Note the increased variability in force trajectories and $d F / d t$ (middle and bottom) and irregularities in the falling part of the force profile.
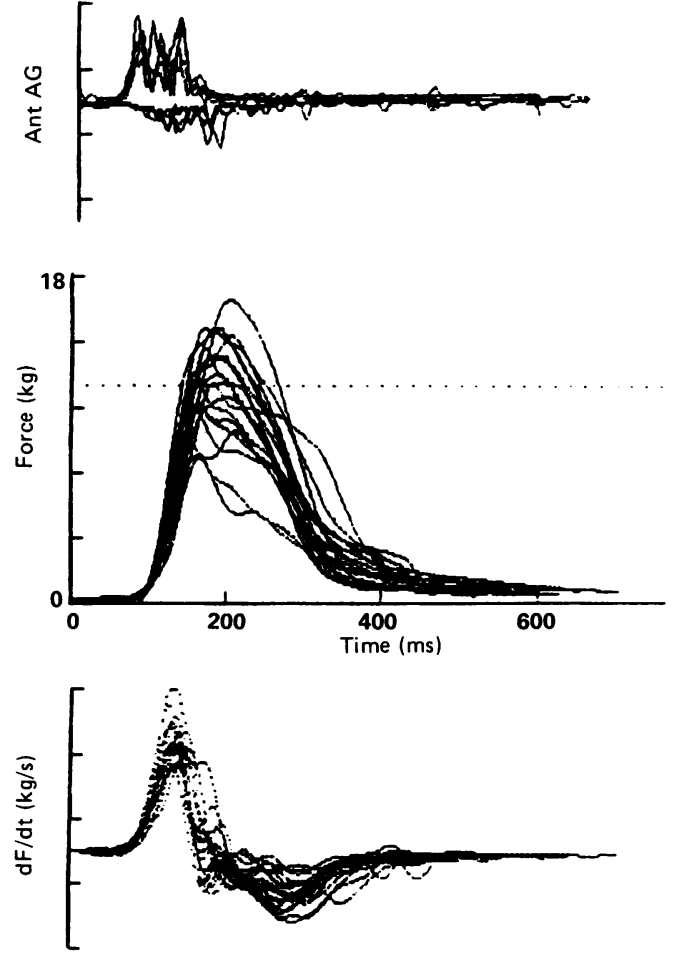
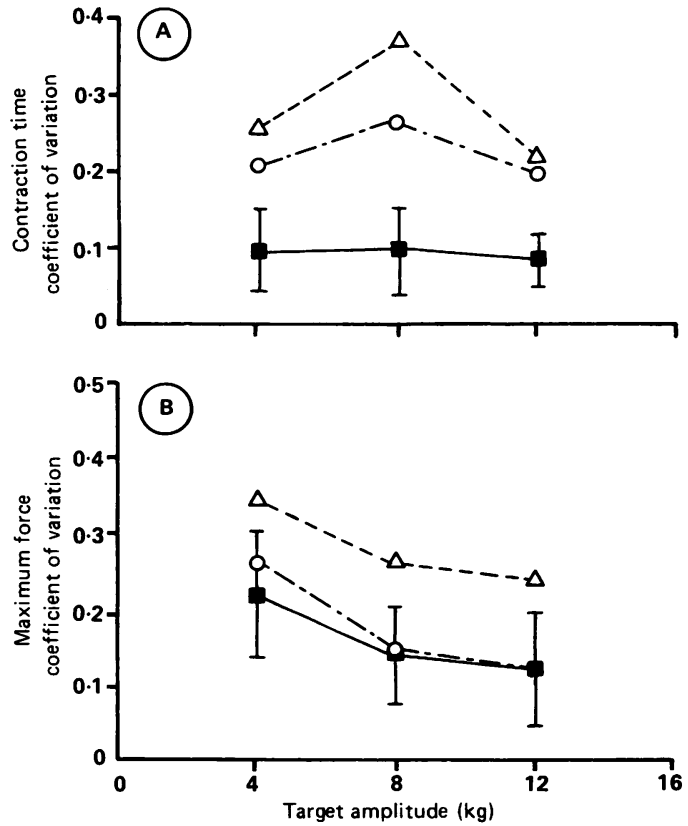

Figure 6 Mean coefficients of variation of force rise time $(A)$ and maximum force $(B)$ in the three groups: normal (squares), Group 1 patients (triangles), Group 2 patients (circles), with $\pm 2 S D$ indicated for normal subjects. 
Figure 7 Force responses produced by Group 2 patients $(A)$ with multiple bursts of EMG at approximately $10 \mathrm{~Hz}$ and $(B)$ with prolonged EMG activity fractionated into bursts but not separated by silent periods. (Top) Typical segmented $(A)$ force responses and smoother $(B)$ force responses; (middle) $d F / d t ;$ and (bottom) single contraction with EMG superimposed. Target amplitude $(12 \mathrm{~kg})$ is shown by the dotted line.
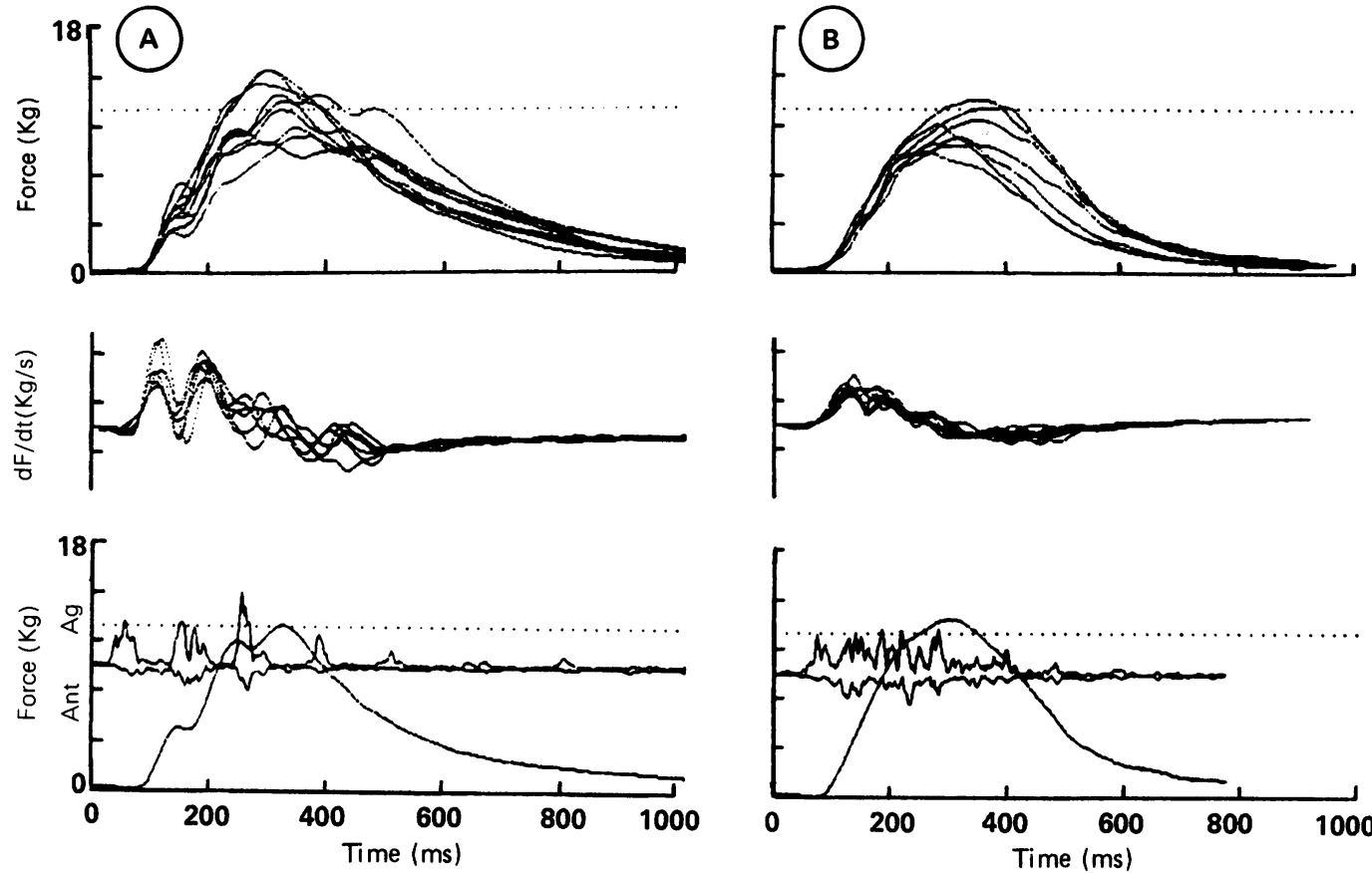

Discussion

MOST-RAPID CONTRACTIONS OF NORMAL SUBJECTS

Normal subjects performing fast isometric contractions to match a visual target of different amplitudes scale the speed of contraction to the target amplitude in such a way that the contraction time is kept relatively constant. This phenomenon has been called the "speed control" strategy" or "pulse control" regulation. ${ }^{10}$ It is accomplished by proportionally scaling the magnitude of the agonist EMG burst with target amplitude, while duration of the burst remains relatively constant. The duration of the agonist burst determines the time to reach the target. ${ }^{11}$

Rapid goal-directed isometric muscle contractions or isotonic limb movements require forces acting in opposite directions which are provided by activation of agonist and antagonist muscles. Agonist muscles produce force in the required direction (or, in the isotonic paradigm, accelerate the limb) whereas antagonists terminate the movement, or the

Figure 8 Analysis of the initial speed of contraction in two Group 2 subjects who produced clearly segmented force responses. (A) Each triangle is maximum $d F / d t$ during the first segment, versus force reached at the end of that segment. Solid line is the regression line for the equivalent data in normal subjects. Note that $d F / d t$ would be almost normal if the target were the force level reached at the end of the first segment. ( $B)$ When $d F / d t$ is plotted instead against the maximum force ultimately achieved, the deficit in $d F$ dt can be quantified.

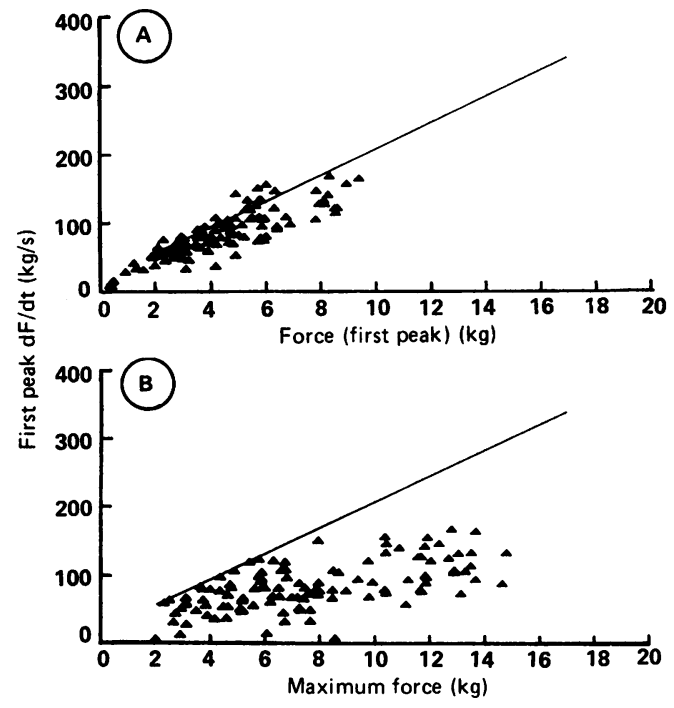

rise time of force trajectory. ${ }^{11} 12$ In fast muscle contractions, both sequential ${ }^{12}{ }^{13}$ and synchronous $^{914}$ patterns of EMG activity in flexor and extensor muscles have been reported. In this study we observed both patterns in a group of normal subjects, with each subject consistently using one pattern. The two EMG patterns produced differently shaped force pulses, with faster decline of force to baseline (more symmetric and narrow pulses) in subjects who used reciprocal activation (fig $1 \mathrm{~A}$ ), rather than coactivation (fig 1B). These differences can be seen most clearly in the plots of the negative portion of $\mathrm{dF} / \mathrm{dt}$.

\section{MOST-RAPID CONTRACTIONS IN \\ PARKINSONIAN PATIENTS}

Parkinsonian patients had difficulty producing rapid single contractions of a given amplitude, despite treatment with medication which improved their everyday motor performance. We observed a spectrum of disordered responses rather than one common pattern for all patients. The large variability in performance of voluntary movements, not only between patients, but also between trials in the same patient, is well known from clinical experience. Three patients were able to produce fast contractions, but occasionally their responses were abnormal. The increased variability of force trajectory parameters such as force rise time and peak force, in comparison with the normal group, provides a quantitative measure of changes in motor performance. These changes might be related to the early stage of $\mathrm{PD}$, as two out of three of these patients had been diagnosed within the past three years. Thus our data suggest that the relatively frequent deviations from a stereotyped movement pattern are a more sensitive indicator of Parkinsonian pathology than average kinematic values, a hypothesis which should be tested on a larger sample of patients.

Our results suggest that patients' ability to 
Figure 9 (A) Complete. EMG-force relationship in a normal subject

(solid + open circles) can be predicted based on

regression line (solid line) fitted to data from lower amplitude contractions (solid circles). ( $B, C)$

Variation of first agonist

EMG burst magnitude with force in a patient producing segmented contractions. The integrated agonist EMG burst is plotted (B) against the peak force reached at the end of the first force segment, and (C) against maximal force. $A$ regression line fitted to the data in (B) defines the EMG-force relation for fast (singlestep) movements; this "calibration" allows us to demonstrate the deficit in actual EMG below what would be required (dashed line) to reach the target in a single step (C).
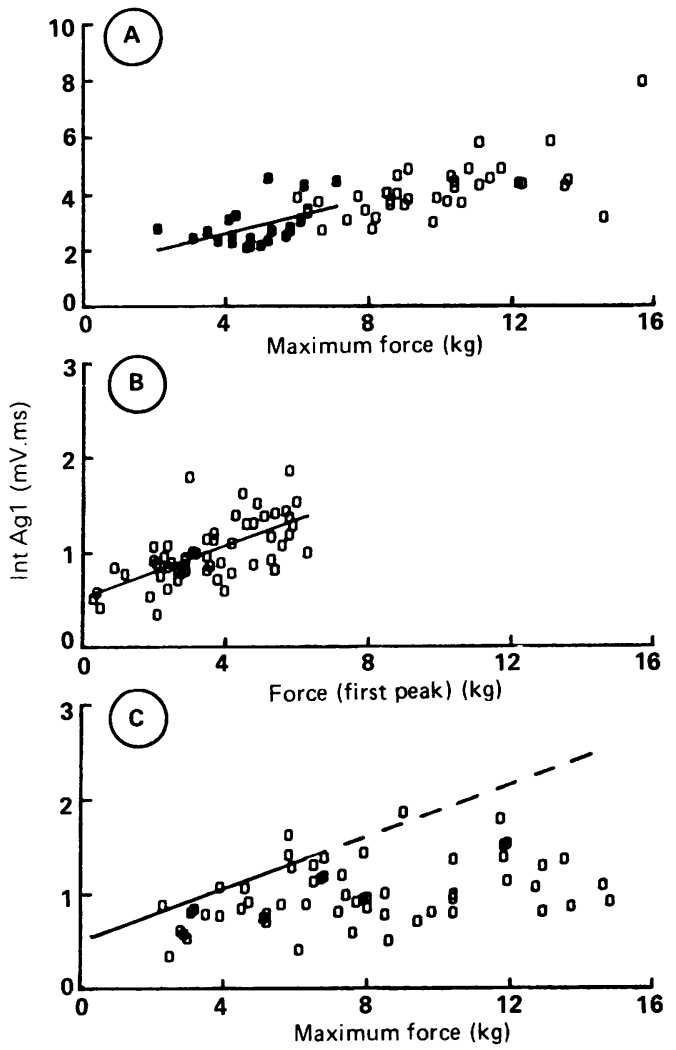

perform fast dynamic force pulses is correlated with their maximum voluntary tonic force production, an observation which has not been previously reported. Although our sample consists of only 11 patients, the probability that the same three patients would turn out both to have the largest MVC and to produce rapid, but variable, force pulses, is approximately 0.006 , which suggests that this correlation did not occur by chance. One explanation for such a correlation may be that in Parkinson's disease, progressive impairment in ability to increase motor unit firing rate ${ }^{15}$ ultimately affects both tonic and dynamic contractions. It is noteworthy that the three patients with the highest MVC were capable of fast, albeit variable, performance of force pulses, since very rapid contractions, requiring high instantaneous firing rates, ${ }^{16} 17$ may be affected even when tonic maximum voluntary contractions, requiring lower discharge rates, ${ }^{18}$ are not.

Force contractions of the remaining eight patients were abnormally slow; the force rise time increased substantially with target amplitude. This was because the first agonist EMG burst and, consequently, the rate of development of force, were smaller than required for a fast movement to the target (fig 8B, 9C). Similarly, other investigators have found the size of the initial EMG burst to be reduced, ${ }^{46}$ but that it could be increased somewhat in order to produce the larger movements. ${ }^{7}$ It should be emphasised that the relative increase of both contraction speed $(\mathrm{dF} /$ dt) and, to a lesser extent, the size of the first agonist EMG burst, with target amplitude, is the same for patients and normal subjects, as was also reported by Berardelli et $a l^{7}$ in their studies of elbow flexions of $15^{\circ}$ and $60^{\circ}$. Although, in general, patients are able to increase
EMG burst area and $\mathrm{dF} / \mathrm{dt}$ with target amplitude, they apparently cannot produce a larger EMG burst with a small target to yield a faster contraction.

SMU firing characteristics and rapid isometric contractions

The reason for the reduction in size of the first agonist burst in PD is not clear. If the amplitude of the EMG burst is proportional to the total number of single motor unit (SMU) discharges during the burst, one can speculate that burst reduction in PD reflects the inability to produce a sufficient number of SMU discharges. Physiologically, increase in discharge number can be achieved by recruitment of additional SMUs and/or by increasing the firing rate of already activated SMUs. Abnormalities of burst amplitude in patients with Parkinson's disease must then be related to impairment of one or both of these mechanisms.

Studies of SMU behaviour in different motor acts such as slow ramp contractions, ${ }^{161719-21}$ ballistic contractions, ${ }^{1617}$ tonic contractions, ${ }^{18}$ and fast alternating voluntary movements ${ }^{22}$ have shown remarkable flexibility in adjustment of SMU discharge characteristics according to motor task requirements. In slow ramp contraction, for example, recruitment and increase of SMU firing rates occur progressively as muscle force increases. By contrast, in fast muscle contractions, SMUs are recruited almost instantaneously, with repetitive discharges at high instantaneous frequencies $(50-100 \mathrm{~Hz})$ early in the burst, followed by a drop later in the burst. ${ }^{16} 17$

We have shown that patients' first agonist EMG burst often does not generate enough force to reach the desired target. To reach the target, the muscle must be activated longer, which may be accomplished either by repetitious EMG bursts (fig 7A) or by a prolonged continuous discharge (fig 7B). These patterns of muscle activation presumably reflect underlying changes in motor unit firing behaviour. Abnormal SMU firing properties found in patients with PD include a delay in recruitment of SMUs, abnormally low discharge frequencies, synchronous bursting of different SMUs, and lapses in SMU firing rates. ${ }^{1523}$ Treatment with levodopa has led to improvement in firing characteristics of SMUs, ${ }^{1523}$ increase in size of the first agonist burst, and faster contraction time and movement velocity..$^{5-7}$

The spectrum of abnormalities in force responses and in EMG reported in our study must be explained by abnormalities of SMU behaviour in PD. In the three patients who were able to produce fast contractions, SMUs must have fired normally in most of the recorded trials, resulting in agonist burst amplitudes proportional to target amplitude. But in PD SMUs usually cannot be driven to continuously discharge at higher frequencies as contraction force increases. Instead, they switch to a slow, synchronised discharge pattern ${ }^{23}$ with SMU burst repetition rate and tremor frequency increasing as force increases ${ }^{24}$ 
and producing the prolonged, irregularly fragmented agonist EMG bursts observed in some of our patients. Although none of these patients had resting tremor at the time of testing, they often had oscillations visible in their force trajectories, most clearly seen in patients with segmented responses. In patients unable to obtain high instantaneous SMU discharge rates within a single burst, sequential EMG bursts (fig 7) reflecting the maximum SMU firing rate may be an important mechanism which allows them to build up force to the target level.

\section{Summary and possible mechanisms}

Our observations of rapid force pulses may be summarised as follows: 1) Both alternating (triphasic) and synchronous EMG bursts in agonists and antagonists were observed, in both control subjects and patients with mild PD;2) The underlying pathology of PD leads to an inability to make stereotyped responses on command and an inability to generate an EMG burst of sufficient intensity to smoothly and quickly accomplish an isometric motor task. This reduction of the level of muscle activation does not affect the ability to increase muscle activity or contraction speed with increasing target amplitude, suggesting that there may be different neural mechanisms involved in setting the original intensity of muscle activation and adjusting the EMG response to increased task requirements. Two recent lines of research suggest candidates for these mechanisms.

Watts et $a l^{25}$ and Mandir et $a l^{26}$ have shown abnormalities of supplemental motor area (SMA) and primary motor cortex (MI) function in macaque monkeys with MPTPinduced Parkinsonism. Following methylphenyl-tetrahydropyridine (MPTP) administration, monkeys showed prolonged reaction times, prolonged variable movement times, and disorganised EMG activity in a simple motor task. Discharge of "preparatory set" cells in the SMA normally increases to a peak just before the burst of MI cell activity which in turn drives the lower motor neurons (SMUs). After MPTP, SMA neurons lost directional specificity, and the timing characteristics of SMA "preparatory set" cells were altered, possibly playing a role in the prolonged reaction times. In addition, those cells in MI best related to movement initiation were disorganised, demonstrating lower peak discharge frequencies and prolonged latencies from onset of discharge to motion onset. Also, there were increased latencies between the task "Go" signal to firing of "Go"-related neurons, and from these "Go"-related neurons to movement onset. These data suggest mechanisms for the slow onset and variability of Parkinsonian movements, with retention of the ability to scale responses.

Delwaide et $a l^{27}$ have recently shown abnormal function in the reticulospinal pathways descending to the spinal cord in patients with Parkinson's disease. This abnormality was illustrated by reduced facilitation of the $H$ reflex as a result of a startle reaction in patients compared with controls. Since SMU firing is generated by descending activity not only in the corticospinal tract, but also in other descending tracts such as the reticulospinal, deficits in these other tracts, along with lower firing rates in primary motor cortex, may account for the systematic undershooting of task targets which we and others have observed. Whether patients can be taught to increase their corticospinal drive to compensate for deficiencies in other descending pathways, in a sense relearning simple motor tasks, is a question for further research.

1 Flowers K. Ballistic and corrective movements on an aiming task. Neurology 1975;25:413-21.

2 Hallett M, Shahani BT, Young RR. EMG analysis of stereotyped voluntary movements at the elbow in patients with Parkinson's disease. J Neurol Neurosurg Psychiatry 1977;40:1129-35.

3 Wiesendanger $M$, Ruegg DG. Electromyographic assessment of central motor disorders. Muscle and Nerve $1978 ; 1: 407-12$.

4 Hallett M, Khoshbin S. Physiological mechanism of bradykinesia. Brain 1980;103:301-14

5 Baroni A, Benvenuti F, Fantini L, Pantaleo T, Urbani F. Human ballistic arm abduction movements: Effects of L-dopa treatment in Parkinson's disease. Neurology Cleveland / 1984;34:868-76.

6 Dick JPR, Rothwell JC, Berardelli A, et al. Associated postural adjustments in Parkinson's disease. J Neurol postural adjustments in Parkinson's
Neurosurg Psychiatry 1986;49:1378-85.

7 Berardelli A, Dick JPR, Rothwell JC, Day BL, Marsden $\mathrm{CD}$. Scaling of the size of the first agonist EMG burst during rapid wrist movements in patients with Parkinson's disease. J Neurol Neurosurg Psychiatry 1986, 49:1273-9.

8 Watts RL, Wiegner AW, Young RR. Elastic properties of muscles measured at the elbow in man: II. Patients with Parkinsonian rigidity. J Neurol Neurosurg Psychiatry 1986;49:1177-81.

9 Freund $\mathrm{H}-\mathrm{J}$, Budingen $\mathrm{HJ}$. The relationship between speed and amplitude of the fastest voluntary contractions of human arm muscles. Exp Brain Res 1978;31:1-12.

10 Gordon J, Ghez C. Trajectory control in targeted force impulses. II. Pulse height control. Exp Brain Res 1987; 67:241-52.

11 Wierzbicka MM, Wiegner AW, Shahani BT. Role of agonist and antagonist muscles in fast arm movements in man. Exp Brain Res 1986;63:331-40.

12 Ghez C, Gordon J. Trajectory control in targeted force impulses. I. Role of opposing muscles. Exp Brain Res 1987;67:225-40.

13 Gordon J, Ghez C. EMG patterns in antagonistic muscles during isometric contraction in man: relations to response dynamics. Exp Brain Res 1984;55:167-71.

14 Hefter H, Homberg V, Lange HW, Freund H-J. Impairment of rapid movement in Huntington's disease. Brain 1987;110:585-612.

15 Milner-Brown HS, Fisher MA, Weiner WJ. Electrical properties of motor units in Parkinsonism and a possible properties of motor units in Parkinsonism and a possible Psychiatry 1979;42:35-41.

16 Desmedt JE, Godaux E. Ballistic contractions in man: Characteristic recruitment pattern of single motor units of the tibialis anterior muscle. $J$ Physiol (Lond) 1977;264: 673-93.

17 Desmedt JE, Godaux E. Ballistic contractions in fast or slow human muscles: discharge patterns of single motor units. J Physiol (Lond) 1978;285:185-96.

18 Bellemare F, Woods JJ, Johansson R, Bigland-Ritchie B. Motor-unit discharge rates at maximal voluntary contractions of three human muscles. $J$ Neurophysiol 1983;50: 1380-92.

19 Milner-Brown HS, Stein RB, Yemm R. The orderly recruitment of human motor units during voluntary isometric contractions. J Physiol (Lond) 1973;230:359-70.

20 Milner-Brown HS, Stein RB, Yemm R. Changes in firing rate of human motor units during linearly changing rate of human motor units during linearly changing voluntary

21 Freund $\mathrm{H}-\mathrm{J}$, Budingen $\mathrm{HJ}$, Dietz V. Activity of single motor units from human forearm muscles during voluntary isometric contractions. $J$ Neurophysiol 1975;38:933-46.

22 Logigian EL, Wierzbicka MM, Bruyninckx F, Wiegner AW, Shahani BT, Young RR. Motor unit synchronization in physiologic, enhanced physiologic, and voluntary tremor in man. Ann Neurol 1988;23:242-50.

23 Petajan JH, Jarcho LW. Motor unit control in Parkinson's disease and the influence of levodopa. Neurology 1975; 25:866-9.

24 Dietz V, Hillesheimer W, Freund H-J. Correlation between tremor, voluntary contraction, and firing pattern of motor units in Parkinson's disease. $J$ Neurol Neurosurg Psychiatry 1974;37:927-37.

25 Watts RL, Mandir AS, Montgomery EB Jr. Abnormalities of supplementary motor area task-related neuronal 1990;5(Suppl 1):78.

26 Mandir AS, Watts RL, Buchholz SR, Montgomery EB Jr. Changes in primary motor cortex neuronal activity associated with increased reaction time and movement time in MPTP parkinsonism. Movement Disorders 1990; 5(Suppl 1):77.

27 Delwaide PJ, Pepin JL, Maertens de Noordhout A. La rigidité parkinsonienne: aspects cliniques et physiopathologiques. Rev Neurol (Paris) 1990;146 (in press). 